http://jmscr.igmpublication.org/home/

ISSN (e)-2347-176x ISSN (p) 2455-0450

crossref DOI: https://dx.doi.org/10.18535/jmscr/v8i1.140

Journal Of Medical Science And Clinical Research

IGM Publication

An Official Publication of IGM Publication

\title{
Reporting a Clinicoradiological Diagnostic Dilemma: Gossypibioma - A Case Report
}

\author{
Authors \\ Dr Nithi Doley*, Dr Ruby Chattopadhyay \\ Indian Naval Hospital Kalyani, Visakhapatnam \\ *Corresponding Author \\ Dr Nithi Doley \\ Department of Pathology, INHS Kalyani, Visakhapatnam, Andhra Pradesh - 530005, India
}

\begin{abstract}
Gossypibioma is a term used to describe a surgical sponge retained at the site of operation and the body's response to the retained foreign body. Gossypibioma is derived from the words: 'Gossypium' which in Latin means cotton and 'boma', a swahili word meaning concealment. It is a surgical mistake which can be avoided if operative theatre records are properly followed. It is very rarely reported because of the the medicolegal implications and criticism by the mass media. Gossypibioma presents as a clinical and radiological diagnostic dilemma because of its variable and vague clinical presentations particularly in longstanding cases and imaging may mimic intra-abdominal abscess or neoplasm. Gossypibioma may also present as an intra-abdominal soft tissue swelling or localised pain abdomen particularly following a surgery. Most of the cases of Gossypibioma are usually detected within few days after surgery, but some may remain undetected for many years. A case of 27 year old female was referred to our centre with a complaint of abdominal fullness since one year after delivery of her child by caesarean section. After clinical and radiological correlation a differential diagnosis of Gastrointestinal stromal tumour, Desmoid tumour, Gossypibioma was made. An exploratory laparotomy was performed, the mass was resected and tissue was sent for histopathological examination to confirm the diagnosis of Gossypibioma.

Keywords: Retained surgical mop, gossypibioma, retained foreign body, post surgery abdominal swelling.
\end{abstract}

\section{Case Report}

\section{Introduction}

A 27 year old female was referred to our hospital with a complaint of abdominal swelling for the last one year. She had a history of delivery of her second child by caesarean section one year back. The patient complained of swelling and abdominal discomfort after delivery of her second child. She also complained that the swelling and abdominal discomfort increased in the last 3 months. There was no history of fever, chills, nausea, vomiting, constipation, dysuria or weight loss of body. The medical and surgical history was insignificant except for delivery of 02 living issues by caesarean section. The younger child was delivered by caesarean section one year back. Her surgical records show that, it was a emergency caesarean section and blood transfusion was done due to intraoperative blood loss.

\section{Clinical Examination}

General examination of the patient revealed vitals were within normal limits. Abdominal examination revealed a surgical scar. A firm mass 


\section{JMSCR Vol||08||Issue ||01||Page 868-870||January}

with smooth surface, round, non tender of size approximately $8 \mathrm{~cm} \times 7 \mathrm{~cm}$ was felt in the periumbilical and left lumbar region.

\section{Diagnostic Investigations}

Laboratory tests, USG abdomen and CT scan abdomen were carried out.

The laboratory parameters were within normal limit.

On USG an ill defined hypoechoiec mass measuring approximately 57 × 87 x $71 \mathrm{~mm}$ was seen in left paramidline location. Prominent shadowing over the mass was also noted.

On CT scan abdomen a differential diagnosis of GIST and Desmoid tumour was made. Exploratory Laparotomy was carried out. The mass was excised and submitted for Histopathological examination.

The patient underwent Exploratory Laparotomy for the abdominal mass. The mass was excised and submitted for Histopathological examination. Post operative period was uneventful and the patient recovered well. The patient was discharged on postoperative day 15 and was advised for follow up.

\section{Gross Examination}

revealed an encapsulated cystic cavity containing a surgical swab surrounded by messentric fat.

Microscopic Examination revealed foreign body granuloma comprising of multinucleated giant cells, dense lymphomononuclear inflammatory infiltrate along with foci of necrosis and hemorrhage. [Pic 1,2,3,4

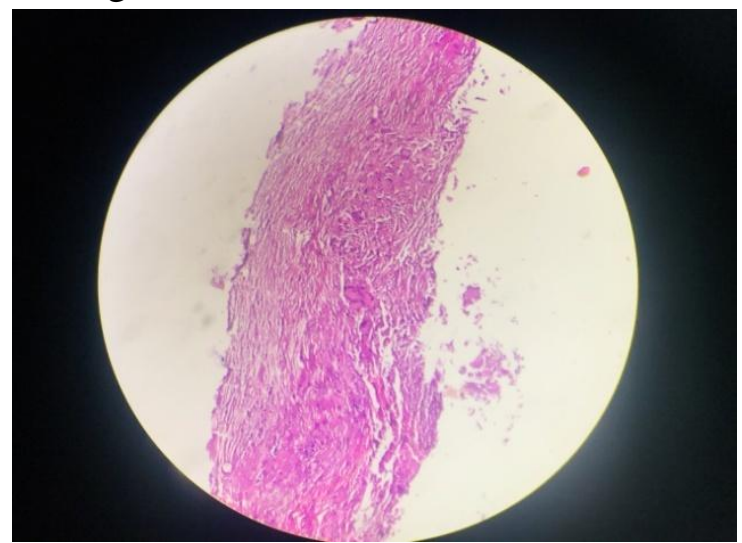

Pic 1: Photomicrograph showing foreign body reaction $(4 \mathrm{x})$

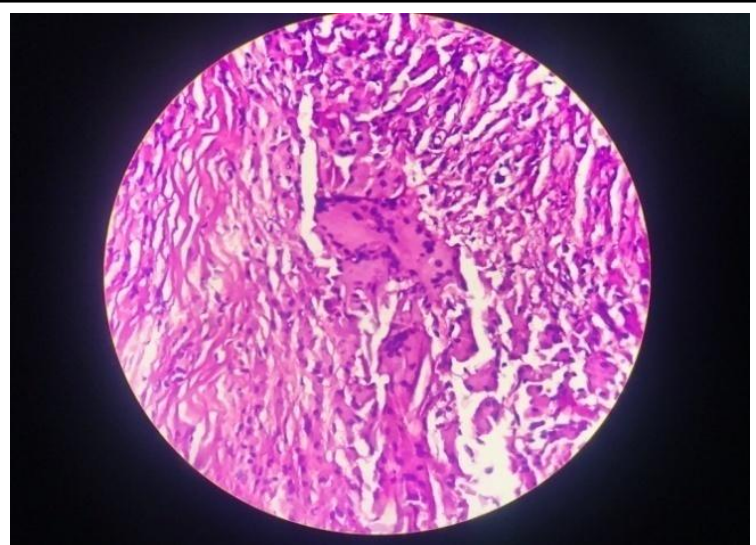

Pic 2: Photomicrograph showing multinucleated giant cells and lymphomononuclear inflammatory infiltrate in the cyst wall (40x).

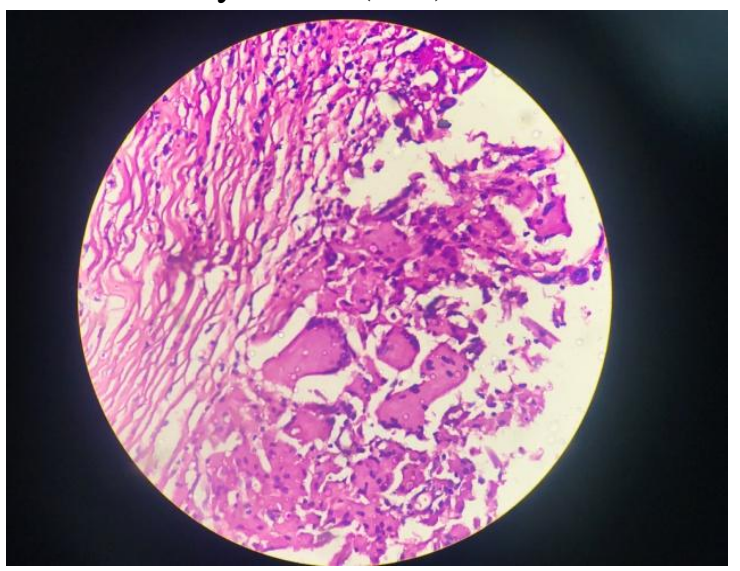

Pic 3: Photomicrograph showing foreign body granuloma in the cyst wall (40x)

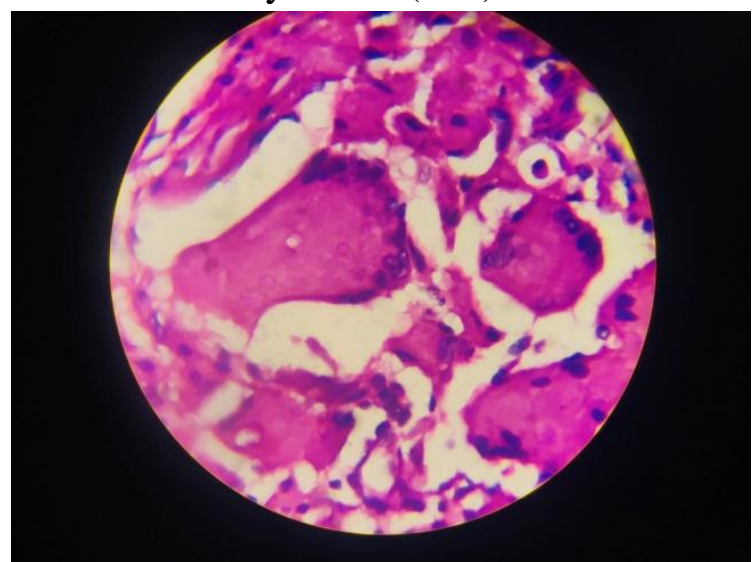

Pic 4: Photomicrograph showing multinucleated giant cell (100x)

\section{Discussion}

ICD 10 [International Statistical and related health problems for classification of Diseases] is the classification given to retained foreign body/object $^{[1]}$. The first case of Gossypibioma was reported by Wilson. Gossypibioma is most commonly encountered in the intraperitoneal 
cavity. But there are some uncommon sites where it can be detected such as the CNS, breast, chest and extremities ${ }^{[2]}$. Gossypibioma can causes very serious medical complications and may lead to medico-legal lawsuits. Gossypibioma is under reported as many of the cases are not reported because of legal implications. The incidence varies from $1 / 100$ to $1 / 3000$ for all surgical interventions $^{[3]}$. Many high risk factors have been identified for Gossypibioma which includes high body mass index, urgent surgeries, prolonged and difficult operative procedures, surgeon fatigue, change of surgical and nursing staff, unmeticulous counting of surgical swabs and lastly human error $^{[4]}$. Patients usually present with non-specific symptoms such as abdominal swelling, vague abdominal pain etc. They may also present with acute symptoms such as abdominal distension and pain, obstruction, sepis or pseudotumour symptoms $^{[5]}$. Pathologically, two types of reactions may occur - one is an aseptic encysted granuloma and the other is frank abscess with or without secondary infection ${ }^{[6]}$. Radiopague swabs are surgical swabs impregnated with dye such as barium sulphate which can be detected with X ray. Thus the use of radioopague surgical swabs will help in the detection of any intraoperatively retained surgical swab. Hence it should be made mandatory that all surgical swabs should undergo radiological screening besides meticulous counting before closure ${ }^{[5]}$. It is also advised that swabs which are held with forceps should be used to prevent intraoperative loss of swabs ${ }^{[4]}$. Newer technologies such as electronic computer assisted sponge counting system and radiofrequency identification of sponges will help reduce the incidence of retained surgical objects to a great extent $^{[1]}$.

\section{Conclusion}

Gossypibioma or retained foreign body (RFB) should be considered in the differential diagnosis of any patient who presents with pain abdomen, infection or palpable mass with a history of prior operation. The diagnosis of Gossipibioma or RFB by clinical suspicion or imaging modality is confirmed by excision and histopathological examination.

\section{Reference}

1. G V Santhosh Kumar, Subhash Ramani, Abhishek Mahajan, Nikshita Jain, Rachel Sequeira, and Meenakshi Thakur, "Imaging of retained surgical items: A pictorial review including new innovations", Indian J Radiol 2017 Jul-Sep; 27(3): 354-361.

2. Seema Chopra, Vanita Suri, Pooja Sikka, and Neelam Aggarwal, "A Case Series on Gossypiboma - Varied Clinical Presentations and Their Management", J Clin Diagn Res. 2015 Dec; 9(12): QR01-QR03. Published online $2015 \quad$ Dec 1. doi:10.7860/JCDR/2015/15927.6978.

3. Yun-Xiao Lv, Cheng-Chan Yu, Chun-Fang Tung, and Cheng-Chung $\mathrm{Wu}^{\mathrm{\prime}}$ ' Intractable duodenal ulcer caused by transmural migration of gossypiboma into the duodenum - a case report and literature review", BMC Surg. 2014; 14: 36. Published online 2014 Jun 10. doi:10.1186/1471-2482-14-36.

4. Vamsi Krishna, D Bharath kumar "Intraluminal migration of Gossypibioma" International Journal of Surgery Case Reports. Volume 47, 2018, Pages 61-63.

5. Sarath Chandra Sistla, Ananthakrishnan Ramesh, Vilvapathy Sengutuvan Karthikeyan et al., "Gossypiboma Presenting as Coloduodenal Fistula - Report of a Rare Case With Review of Literature",Int Surg. 2014 Mar-Apr; 99(2): 126-131.

6. Temidayo Og undiran, Omobolaji Ayandipo, Adenike Adeniji-Sofoluwe et al. "Gossypiboma: complete transmural migration of retained surgical sponge causing small bowel obstruction", BMJ Case Rep. 2011; 2011: bcr0420114073. Published online 2011 Jun 30. doi:10.1136/bcr.04.2011.4073. 